\title{
Mode transitions and wave propagation in a driven-dissipative Toda-Rayleigh ring
}

\author{
E. del Río, ${ }^{1, *}$ V. A. Makarov, ${ }^{2}$ M. G. Velarde, ${ }^{2,3, \dagger}$ and W. Ebeling ${ }^{4}$ \\ ${ }^{1}$ ETSI Aeronauticos, Universidad Politecnica de Madrid, Plaza Cardenal Cisneros, 3, Madrid 28040, Spain \\ ${ }^{2}$ Instituto Pluridisciplinar, Universidad Complutense, Paseo Juan XXIII, 1, Madrid 28040, Spain \\ ${ }^{3}$ International Center for Mechanical Sciences (CISM), Palazzo del Torso, Piazza Garibaldi, Udine 33100, Italy \\ ${ }^{4}$ Institut für Physik, Humboldt-Universität, Invalidenstrasse, 110, Berlin 10115, Germany \\ (Received 10 May 2002; revised manuscript received 21 January 2003; published 19 May 2003)
}

\begin{abstract}
A circular lattice (ring) of $N$ electronic elements with Toda-type exponential interactions and Rayleigh-type dissipation is used to illustrate wave formation, propagation, and switching between wave modes. A methodology is provided to help controlling modes, thus allowing it to realize any of $(N-1)$ different wave modes (including soliton-type modes) and the switching between them by means of a single control parameter.
\end{abstract}

DOI: 10.1103/PhysRevE.67.056208

PACS number(s): 05.45.- a, 84.30. $-\mathrm{r}, 45.50 .-\mathrm{j}$

\section{INTRODUCTION}

Cyclic global behavior, with intermediate jumping or switching between different modes of operation (in general of complex form), is known to occur in various fields of science and engineering and particularly, in biology, neurobiology, and biochemistry [1]. An example is the versatile and elegant locomotion of animals with efficient gait switching that has stimulated efforts to mimick it with artificial walking machines [2-5]. To solve the problem of pattern formation and robust transition among several types of gaits the use of oscillatory neural networks with different architectures has been purposed $[3,6]$. In fact, most animal gaits possess a degree of symmetry and universal features not far from the behavior of rings of coupled oscillators [7-9]. Then transitions between different gaits can be modeled as symmetry breaking bifurcations, leading to the switch between different activity patterns in a ring [8-10]. In general, in a ring lattice possessing several operational regimes (multistable modes) switching between them can be achieved with a suitable resetting of the phases of all units. Different robust methods to control operational modes have been proposed. For instance, in Refs. $[6,4]$ the control of a hexapode robot by means of patterns generated by reaction-diffusion cellular neural networks is discussed and implemented.

On the other hand, coupled map lattice models have been used to study complex spatio-temporal systems $[11,12]$. In this way, the evolution of ring lattice systems has been considered in Refs. [13-15], investigating, e.g., spatiotemporal periodic traveling waves and their behavior depending on initial conditions and values of control parameters [13]. The behavior of fronts near the boundary of parametric instability has been investigated in Ref. [14] and a connection between a periodic solution, spatiotemporal intermittency, bifurcation behavior, and coexisting attractor is studied in [15], for coupled sine circle and coupled logistic map lattices.

Using analog circuits is a well-known $[16,17]$ approach in the study of the behavior of the nonlinear dynamics of rings made up of coupled oscillators. In this case, we have a true

\footnotetext{
*Email address: edelrio@aero.upm.es

${ }^{\dagger}$ Email address: velarde@ fluidos.pluri.ucm.es
}

physical system exhibiting robustness and tolerance to (low) noise level and a slight parameter mismatch. Yet, a system can show diversity in behavior due to noise [18] or due to detuning of the oscillators of the ring [19]. The dynamics of a ring of coupled oscillators has been considered in Ref. [20] for the experimental observation of emergent cyclic behavior associated to a fast periodic rotating wave.

Toda [21] provided exact analytic solutions, including solitons, for a one-dimensional (1D) Hamiltonian lattice (chain) with exponential interactions between the elements. Subsequently, other (conservative) solitons bearing chains were proposed to study nonlinear signal evolution and transmission. Electronic implementations of such chains are unavoidably dissipative, which may drastically alter the initially expected behavior. As dissipation leads to energy loss [22-26], energy injection is necessary to (steadily) maintain the evolution of the system. We therefore study here the role of dissipation and controlled energy pumping in the formation and sustained propagation of different waves, including solitonic waves that are known to propagate in discrete or continuous media [27]. Using as an example, a ring lattice with Toda-type interaction between units and Rayleigh energy dissipation-pumping mechanism [28-30], we show how by means of a simple feedback one can stabilize different activity patterns and achieve robust switching between them. This driven-dissipative Toda lattice also allows autonomous cyclic behavior when modes continuously replace each other in a long, global, overall loop.

Hirota and Suzuki [22] described the first implementation of an electrical circuit of the Toda lattice. However, their implementation was not valid for high amplitude waves. Recently, Singer and Oppenheim [23] proposed a different circuit using junction diodes to model the exponential nonlinearity and double capacitors to obtain appropriate time derivatives for modeling the original, conservative Toda lattice. The resulting circuit was accurate enough to study different soliton effects such as soliton overtaking and collisions. Based on previous results [23,29], in Ref. [30] we proposed an alternative electrical circuit for the investigation of waves and space-time patterns in a Toda-Rayleigh lattice, including dissipative effects and energy input mechanisms. We have shown that, unlike to the Hamiltonian case, the initial conditions lose relevance and the energy input- 


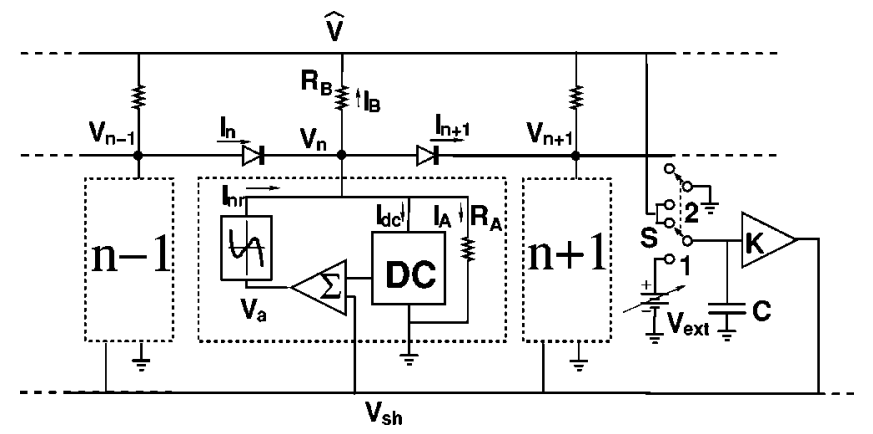

FIG. 1. Block scheme of the Toda-Rayleigh lattice. Each node (dashed box) includes three main blocks: a double capacitor (DC), a nonlinear resistor (its resistance is a nonlinear function of the voltage applied to its terminals), and a voltage adder that shifts the voltage at the nonlinear resistor by a common voltage $V_{\mathrm{sh}} . R_{A}$ is an additional stabilizing high-value resistor. Resistors $R_{B}$ are used to obtain the averaged voltage $\hat{V}$. The circuit at the right, including switch $S$, high capacitance $C$, external battery $V_{\text {ext }}$, and amplifier with variable coefficient $k$, controls the evolution of the ring automatically via a global feedback.

dissipation balance helps to (steadily) maintain a welldefined finite number of different oscillatory solutions. Namely, the Toda-Rayleigh ring possesses $(N-1)$ different nonlinear oscillatory modes or waves (where $N$ is the number of units in the ring) and two nonoscillatory rotational modes. Furthermore, by monitoring a common external voltage, we were able to switch between modes in the circuit allowing selection of waves including solitary waves [30]. Here we significantly extend our previous study, focusing now attention on mode generation, control, and switching mechanisms.

Section II provides details of the electronic implementation of the driven-dissipative Toda-Rayleigh ring. In Sec. III, the stability regions of the $N+1$ modes is provided. We show that the characteristic function of the nonlinear resistor of the units determines the branches in parameter space corresponding to stable wave modes. By tuning a single parameter we can select any mode. Analytical results are compared with the experimental data showing fairly good agreement between the theory and computer experiments. Section IV deals with controlled feedback-induced transitions between wave modes. When parameter values are changed, the lattice successively visits different wave modes in a global long cycle. Finally in Sec. V, we summarize the results obtained, and discuss the relation between the various possible oscillatory modes in a given ring with arbitrary number of units.

\section{DISSIPATIVE TODA-RAYLEIGH LATTICE AND ITS ANALOG CIRCUIT IMPLEMENTATION}

Figure 1 shows a block diagram for the Toda-Rayleigh ring. Reference [30] provides detailed description of all components. To the circuit in Ref. [30], we have added resistors $R_{B}$ to obtain the voltage $\hat{V}$ averaged over all nodes in the ring and an amplifier that can increase or decrease the voltage $V_{\text {sh }}$ supplied to the voltage adders. The amplifier can connect either to a variable voltage source $\left(V_{\text {ext }}\right.$ in Fig. 1$)$ or to the average voltage $\hat{V}$. In the first case, the resistors $R_{B}$ connect in parallel to $R_{A}$, effectively reducing their resistance. In the second case, we add a global coupling with a feedback loop via the amplifier to the voltage adders, and finally to the nonlinear resistors. Due to the high value of $R_{B}$, this global coupling does not affect directly the evolution of the circuit, but instead controls the behavior of the ring via feedback.

From the current-voltage $(I-V)$ relation for a double capacitor (DC) [31] and Kirchhoff's laws, the equation governing the evolution of the circuit is $[23,30]$

$$
\frac{d^{2} V_{n}}{d t^{2}}=\omega_{v}^{2} R_{\mathrm{dc}} I_{\mathrm{dc}}=\omega_{v}^{2} R_{\mathrm{dc}}\left(I_{n}-I_{n+1}+I_{\mathrm{nr}}-I_{A}-I_{B}\right),
$$

where $\omega_{v}^{2} \approx 10^{6}(\mathrm{rad} / \mathrm{s})^{2}$ is a constant depending on the structure of the double capacitor. $I_{\mathrm{nr}}, I_{A}$, and $I_{B}$ are currents, respectively, through the nonlinear resistor and the two linear resistors in the node $n$ (Fig. 1). $I_{n}$ is the current through the junction diode with $I-V$ characteristics:

$$
I_{n}=I_{s} \exp \left(\frac{V_{n-1}-V_{n}}{V_{t}}\right)
$$

where the constants $I_{s}$ and $V_{t}$ depend on the choice of the diode. Depending on the position of the switch, the voltage $\hat{V}$ can be zero (the common point is grounded) or may vary. The current through the nonlinear resistor (block NR in Fig. $1), I_{\mathrm{nr}}$, is a nonlinear function of the applied voltage $V$ $=V_{n}-V_{a}$. The function $I_{\mathrm{nr}}$ is symmetric and cubiclike with three zeros and positive slope at the origin, hence having a region with negative differential resistance, to provide energy pumping and dissipation. A recent monograph by one of the authors [27] treats a similar problem. Further, for numerical simulations we approximate $I_{\mathrm{nr}}$ as

$$
I_{\mathrm{nr}}(V)=\frac{3 \sqrt{3} I_{\max }}{2 \widetilde{V}^{3}}\left(\widetilde{V}^{2}-V^{2}\right) V
$$

where constants $I_{\max }$ and $\widetilde{V}$ are, respectively, the current at the extreme and the coordinate of zero of the function $I_{\mathrm{nr}}$. In our experimental setup, we use $\widetilde{V}=1.45 \mathrm{~V}$ and $I_{\max }$ $=45 \mu \mathrm{A}$. Note that the voltage applied to the nonlinear resistor is a linear combination of the time derivative of the voltage at the node, $V_{n}$, and the "shift" voltage $V_{\text {sh }}[30]$ :

$$
V=\frac{1}{\omega_{v}^{2} R_{\mathrm{dc}} C_{1}} \frac{d V_{n}}{d t}-V_{\mathrm{sh}} .
$$

In our analytical calculations, we use dimensionless quantities denoted by lower case letters:

$$
v_{n}=\frac{V_{n}}{\widetilde{V}_{n}}, \quad i=\frac{I}{I_{\max }}, \quad t^{\prime}=\omega_{v} \sqrt{\frac{I_{\max R} R_{\mathrm{dc}}}{\widetilde{V}} t} .
$$

The rescaled time is $t^{\prime} / t \approx 651.6 \mathrm{~s}^{-1}$.

Equations (1)-(5) give the following equations of motion: 


$$
\begin{gathered}
\ddot{v}_{n}=i_{S}\left(e^{\left(v_{n-1}-v_{n}\right) / v_{t}}-e^{\left(v_{n}-v_{n+1}\right) / v_{t}}\right)+F\left(\tau \dot{v}_{n}-v_{\mathrm{sh}}\right) \\
-a v_{n}-i_{B}, \\
i_{B}= \begin{cases}a v_{n}, & S \text { in position } 1 \\
a\left(v_{n}-\frac{1}{k} v_{\mathrm{sh}}\right), & S \text { in position 2, }\end{cases}
\end{gathered}
$$

with

$$
i_{s}=\frac{I_{s}}{I_{\max }}, \quad a=\frac{\widetilde{V}}{I_{\max } R_{A}} \approx 0.27, \quad \tau=\frac{1}{\omega_{v} C_{1}} \sqrt{\frac{I_{\max }}{\widetilde{V} R_{\mathrm{dc}}}} \approx 0.48
$$

and $F(x)$ has three roots at $x= \pm 1$ and $x=0$. For numerical simulation, we will use $F(x)=(3 \sqrt{3} / 2)\left(1-x^{2}\right) x$. A dot over a quantity denotes time differentiation with respect to $t^{\prime}$.

We also measure the average voltage over the nodes as

$$
\bar{v}=\frac{1}{N} \sum_{n=1}^{N} v_{n}
$$

which, in general, is a function of time with small amplitude variation.

\section{OPERATIONAL MODES AND JUMPS BETWEEN THEM}

With the switch in position 1 (Fig. 1), the current $I_{B}$ is equal to the current $I_{A}$ [Eq. (7) where we assume $R_{A}=R_{B}$ ], so the common shift voltage for all nonlinear resistors is a constant controlled by the voltage of the external battery,

$$
V_{\mathrm{sh}}=k V_{\mathrm{ext}} \text {. }
$$

In this configuration, the ring $(6)$ has $(N+1)$ different stable modes: $(N-1)$ oscillatory modes, and two nonoscillatory rotational modes. The last two correspond to (clockwise or counterclockwise) rotation of the ring as a whole. We assign each mode a mode number $m$ which gives wavelength and wave vector as $N /|m|$ and $2 \pi m / N$, respectively. All modes are nonlinear (anharmonic) oscillations and, hence, nonlinear waves in the ring. The oscillation amplitude and shape also depend on the mode. The modes with negative $m=-1$, $-2, \ldots,-N / 2$ have the same amplitude as the corresponding modes with positive $m=1,2, \ldots, N / 2$ (here we assume $N$ to be even, for odd number of nodes, see Sec. V), corresponding to waves with the same wavelength but propagating in opposite directions. For even $N$, there exists a "special" (optical) mode $m= \pm N / 2$. Both signs define the same oscillatory form with nearest-neighbor units oscillating in antiphase. The two longest wavelength modes (with $m= \pm 1$ ) have the largest amplitude. The amplitude decreases for increasing mode number. Figure 2 shows sample experimental traces for all nodes for the mode $m=1$ (in a six-node ring). For a long enough ring, the $m= \pm 1$ modes become soliton solutions (for voltage difference variables). For two nonoscillatory modes, we assign mode numbers $m= \pm 0$ which have infinite wavelength (or nonoscillating solutions). Here signs show the direction of ring rotation.

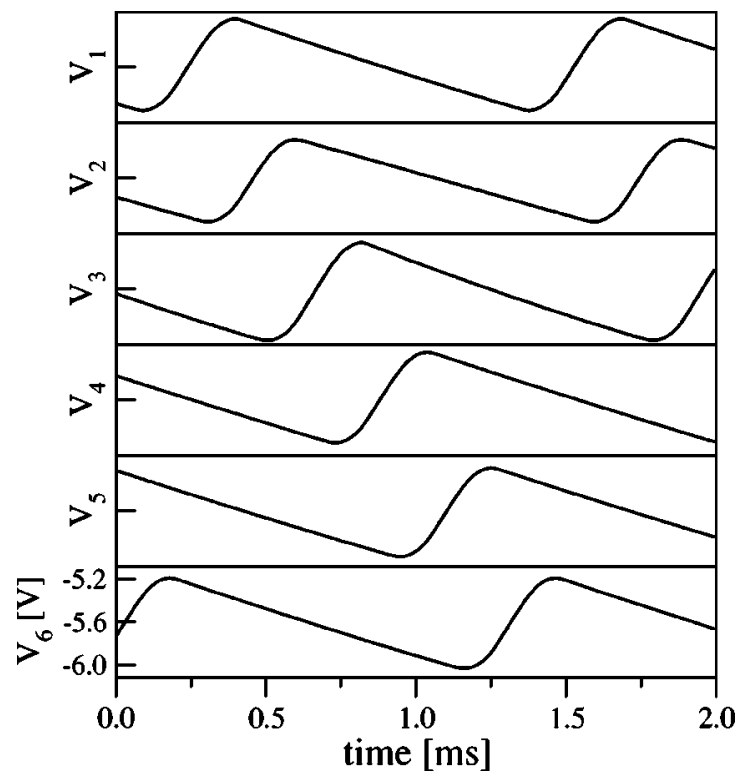

FIG. 2. Oscilloscope traces of the voltages for each node for the $m=1$ mode.

Generally an excitation passing through a node increases (or decreases) its voltage by a value depending on the excitation type ( $\Delta V=\int \dot{V} d t$ need not to be zero). Thus a wave rotating in the ring continuously increases (or decreases for a wave running in the opposite direction) the voltage in the nodes, which eventually saturates the circuit. The voltage adders and the high-value resistors $R_{A}$ (Fig. 1) remedy this problem, as already discussed in Ref. [30].

To describe the mode evolution and switching between modes, we plot the average voltage at a node $\left\langle v_{n}\right\rangle=\langle\bar{v}\rangle$ as a function of the shift voltage $v_{\text {sh }}$ for all allowed modes, mapping the stable solution branch for each mode. Let us find these branches theoretically and then compare the results with experimental observations. For a stationary wave rotating in the ring with period $T$, we have

$$
v_{n}(t)=v(\xi), \quad \xi=t-n \frac{m T}{N}
$$

Hence from Eq. (6), we obtain

$$
\langle\bar{v}\rangle=\frac{1}{a N} \sum_{n=1}^{N}\left\langle F\left(\tau \dot{v}_{n}-v_{\mathrm{sh}}\right)\right\rangle=\frac{1}{a}\left\langle F\left(\tau \dot{v}(\xi)-v_{\mathrm{sh}}\right)\right\rangle_{\xi} .
$$

For two nonoscillatory modes $m= \pm 0[\dot{v}(\xi)=0]$, Eq. (10) gives

$$
\langle\bar{v}\rangle_{ \pm 0}=-\frac{1}{a} F\left(v_{\mathrm{sh}}\right)
$$

The analysis of Eqs. (6) and (11) shows that the domain (branch) $-\infty<v_{\mathrm{sh}}<-v^{*}$ corresponds to the stable $m=-0$ mode (counterclockwise rotation), while $v^{*}<v_{\mathrm{sh}}<\infty$ corre- 


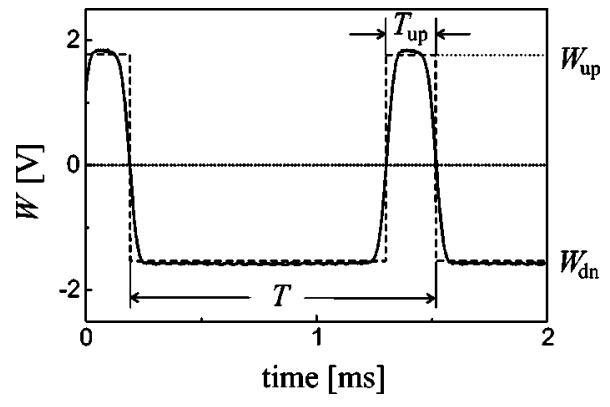

FIG. 3. Voltage at the nonlinear resistor $\left[W \propto w=\tau \dot{v}(\xi)-v_{\mathrm{sh}}\right]$ for the $m=1$ mode (solid line) and step function approximation (dashed line). The wave form is identical to that shown in Fig. 2 for voltages $V_{n}\left(V_{\mathrm{sh}}=1.03 \mathrm{~V}\right)$.

sponds to the stable $m=+0$ mode. Here $v^{*}$ is the coordinate of the maximum of the function $F$. The intermediate domain $-v^{*}<v_{\mathrm{sh}}<v^{*}$ is unstable.

To obtain the dependence of $\langle\bar{v}\rangle_{m}$ (where $m$ is the mode number) on $v_{\text {sh }}$ for an oscillatory mode, we approximate it by a piecewise linear function. Namely, Eq. (10) involves the voltage derivative $\dot{v}(\xi)$, which we approximate by a step function oscillating between two constant values. Experimental observations support this approximation (Fig. 3). Then for a stationary mode with mode number $m$, Eq. (10) gives

$$
\langle\bar{v}\rangle_{m}=\frac{1}{a}\left[\frac{T_{\mathrm{up}}}{T} F\left(w_{\mathrm{up}}\right)+\left(1-\frac{T_{\mathrm{up}}}{T}\right) F\left(w_{\mathrm{dn}}\right)\right],
$$

where $T$ is the oscillation period (which depends on the mode number $m$ ), $T_{\text {up }}$ denotes the time interval when the voltage applied to the nonlinear resistor, $w=\tau \dot{v}(\xi)-v_{\mathrm{sh}}$, is maximal (Fig. 3), and $w_{\text {up }}$ and $w_{\text {dn }}$ are (dimensionless) maximum and minimum voltages at the nonlinear resistor still to be determined. Since the voltage on a node is a bounded function of time, $\int_{0}^{T} \dot{v} d \xi=0$ and

$$
\frac{T_{\text {up }}}{T}\left(w_{\text {up }}+v_{\text {sh }}\right)+\left(1-\frac{T_{\text {up }}}{T}\right)\left(w_{\text {dn }}+v_{\text {sh }}\right)=0 .
$$

The energy is

$$
H=\sum_{n}\left[\frac{\dot{v}^{2}}{2}+i_{s} v_{t} \exp \left(\frac{v_{n-1}-v_{n}}{v_{t}}\right)+a v_{n}^{2}\right],
$$

which corresponds to the dissipationless Hamiltonian ( $F$ $\equiv 0)$. Its time derivative is

$$
\frac{d H}{d t}=\sum_{n} F\left(\tau \dot{v}_{n}-v_{\mathrm{sh}}\right) \dot{v}_{n}
$$

In the stationary state, we have

$$
\int_{0}^{T} \frac{d H}{d t} d t=0
$$

Substituting Eq. (15) into Eq. (16), exchanging integration and summation, and using Eq. (13), we obtain

$$
F\left(w_{\text {up }}\right)=F\left(w_{\text {dn }}\right) \text {. }
$$

The time derivative of Hamiltonian (15) can also be written as

$$
\frac{d H}{d t}=N F\left(w_{\text {up }}\right)\left(w_{\text {up }}+v_{\text {sh }}\right)\left[\frac{q}{N}-\left(1-\frac{q}{N}\right) \frac{T_{\text {up }}}{T-T_{\text {up }}}\right],
$$

where $q(t) \in \mathbb{N}(0 \leqslant q \leqslant N)$ is the number of nodes having $w=w_{\text {up }}$ at time $t_{0}$. In the step function approximation, $\sum \dot{v}_{n}$ is a continuous (smooth) function of time. Hence, voltage jumps of $\left(\tau \dot{v}_{n}-v_{\text {sh }}\right)$ (between $w_{\text {up }}$ and $w_{\text {dn }}$ ) occur synchronously in the ring. The number of units jumping "up" at a time $t$ exactly corresponds to the number of units jumping "down" at this same time, hence $q$ is a constant. From Eq. (18), we obtain

$$
\frac{T_{\text {up }}}{T}=\frac{q}{N} .
$$

Finally, from Eqs. (12), (17), and (19) it follows that

$$
\langle\bar{v}\rangle_{m}=\frac{1}{a} F\left(w_{\mathrm{up}}\right)
$$

where $w_{\text {up }}$ is a positive root of the equation

$$
F\left(w_{\text {up }}\right)+F\left(\frac{N v_{\text {sh }}+q w_{\text {up }}}{N-q}\right)=0,
$$

satisfying the inequalities

$$
\begin{gathered}
w_{-}<w_{\text {up }}<w_{+}, \\
\frac{(N-q) w_{-}-N v_{\mathrm{sh}}}{q}<w_{\text {up }}<\frac{(N-q) w_{+}-N v_{\mathrm{sh}}}{q} .
\end{gathered}
$$

The values $w_{ \pm}$are found from the conditions: $F^{\prime}\left(w_{-}\right)=0$, $F^{\prime \prime}\left(w_{-}\right)<0$ and $F\left(w_{+}\right)=F\left(w_{-}\right)$. The integer $q$ in Eq. (21) corresponds to the mode number:

$$
q= \begin{cases}m, & m \geqslant 0 \\ N+m, & m<0 .\end{cases}
$$

The general solution of Eqs. (20) and (21) is

$$
\langle\bar{v}\rangle_{m}=\frac{1}{a} \Phi\left(v_{\mathrm{sh}}, m\right),
$$

where $\Phi$ is a function only of the shift voltage and mode number, thus defining the stable branches on the plane $\left(\langle\bar{v}\rangle, v_{\mathrm{sh}}\right)$. Figure 4 shows the stable branches $\Phi$, for the six-node ring. We can plot the stable branches of $\Phi$, using either the analytical expression for the function $F$ [see Eq. (3)] or the experimentally measured $I-V$ characteristic [30].

Moving along a branch for a given mode number $q$ (or $m$ ), we come to a point where Eq. (21) has no solution for 


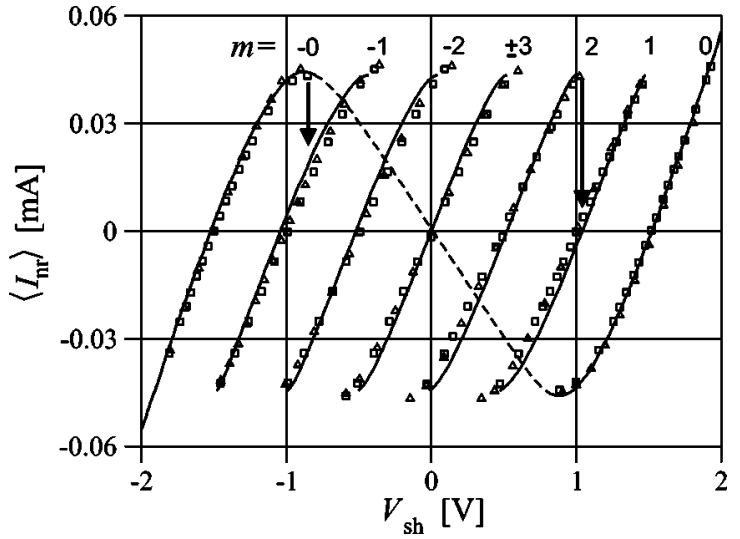

FIG. 4. Mean current across a nonlinear resistor [from Eq. (10) $\left.\left\langle I_{\mathrm{nr}}\right\rangle=\langle\bar{V}\rangle / R_{A}\right]$ vs the shift voltage $V_{\mathrm{sh}}$. Triangles and squares correspond to different experiments with $R_{A}=R_{B}=240 \mathrm{k} \Omega$ and to $R_{A}=R_{B}=120 \mathrm{k} \Omega$, respectively. Solid curves show theoretically predicted [Eq. (24)] stable branches corresponding to different modes. Arrows show possible (experimentally observed) directions of jumps when one mode loses stability (only two arrows are shown).

the given $q$, while for different $q^{\prime} s$ solutions exist. At this bifurcation, the initially excited mode loses stability and a new (stable) mode forms, causing a mode jump. We can estimate the values of the shift voltage $v_{\mathrm{sh}}^{\mathrm{jmp}}$ at which jumps occur using Eq. (21) and the cubic approximation to $F$ :

$$
v_{\mathrm{sh}}^{\mathrm{jmp}}= \begin{cases}\frac{N-3 q}{\sqrt{3} N}, & q \text { increases } \\ \frac{N-3 q}{\sqrt{3} N}+\frac{1}{\sqrt{3}}, & q \text { decreases. }\end{cases}
$$

In the same way we can find the zeros of $\Phi$, which correspond to zero average current through the nonlinear resistors. Setting $\langle\bar{v}\rangle_{m}=0$ in Eq. (20), we get

$$
v_{\mathrm{sh}}^{0}=1-\frac{2 q}{N} .
$$

Accordingly, $\Phi$ has $(N+1)$ different stable branches $(N-1$ for oscillatory modes $0<q<N$, and two for the nonoscillatory modes). Stable branches intersect the $v=0$ axis at $(N-1)$ points defined by Eq. (26). These points uniformly divide the range between the two extreme points \pm 1 which correspond to the nonoscillatory modes [see Eq. (11)]. From Eq. (25), the length (interval in $v_{\mathrm{sh}}$ ) of each stable branch is $1 / \sqrt{3}$, and is independent of $N$. The jumps between modes require overlapping of stable branches for bifurcating values of $v_{\mathrm{sh}}$. Then Eq. (25) gives the minimal number of nodes in the ring $N>3$ to have successful jumps. The more nodes in the ring, the denser the branches of $\Phi$.

To check these theoretical predictions, we studied the influence of the shift voltage $V_{\text {sh }}$ on the evolution of the ring, focusing particularly on the mode formation and stability. For a large enough, negative shift voltage theory predicts the existence of a single solution, the nonoscillatory mode with $m=-0$ ("left" rotation). From Eq. (25), substituting $N=6$ and $q=5$ we estimate the shift voltage $V_{\text {sh }} \lesssim-1.26 \mathrm{~V}$ below which there are no stable oscillatory modes, hence independently of the initial conditions, the mode $m=-0$ is realized. Thus starting from $V_{\mathrm{sh}}=-1.8 \mathrm{~V}$ we ensure that the mode $m=-0(q=6)$ is formed in the ring. Then we slowly increase $V_{\text {sh }}$ to large positive values and follow the mode evolution. Figure 4 shows experimental results for two such runs with different values of $R_{A}$. We observe no difference in the mean current in these two runs, thus confirming the theoretical prediction that branch positions and shapes depend only on the properties of the nonlinear resistor, i.e., on the energy dissipation-pumping mechanism. Furthermore, the experimental results agree with all the stable branches found for the analytically predicted $\Phi$.

As mentioned above earlier, for $N>3$ (in our case $N$ $=6$ ) several modes can be stable for the same shift voltage (Fig. 4). As $V_{\text {sh }}$ increases beyond a critical point [Eq. (25)] and the mean value of the current approaches its maximum $I_{\text {max }}$, the initially excited mode becomes unstable, while some other modes are stable. For example, for $V_{\mathrm{sh}} \gtrsim$ $-0.85 \mathrm{~V}$ the mode $m=-0$ is unstable, but the two other modes with $m=-1$ and $m=-2$ remain stable. Hence, further increase of $V_{\text {sh }}$ leads to a jump to either mode $m=-1$ or $m=-2$ mode. Experimentally we observe both jumps, but for a slowly varying shift voltage (or low perturbation) generally the jump is to a nearest mode, in this case to $m=-1$ as shown in Fig. 4. In other words, the nearest mode in the mode number is the "nearest" in the phase space as well. Subsequently, we jump to $m=-2, m=3, m=2, m$ $=1$, and $m=0$. Similar jumps are observed when the shift voltage decreases from $1.8 \mathrm{~V}$ to $-1.8 \mathrm{~V}$.

We have also found experimentally that decreasing $R_{A}$ by half (from $240 \mathrm{k} \Omega$ to $120 \mathrm{k} \Omega$ ) allows jumps only between the neighboring modes and prohibits jumps to next-nearest neighbors.

Figure 4 shows hysteresis between neighboring modes. Exciting a mode, say with $m=-1$ (using an appropriate value of $v_{\mathrm{sh}}$ ), we can jump to the mode with $m=-2$ by increasing the shift voltage. To return to the $m=-1$ mode, it suffices to decrease the shift voltage to a value where the stable branch for $m=-2$ ends. Note that jumps "forward" and "backward" exhibit hysteresis hence occurring at different values of the shift voltage. From Eq. (25) we can estimate the hysteresis gap $\Delta V_{\mathrm{sh}} \approx 0.42 \mathrm{~V}$ close to the experimental value (Fig. 4). By successive jumps, we can obtain any desired mode.

\section{MODE ROTATION AND MODE CONTROL}

We now use $\bar{v}$ to modulate the shift voltage [the switch in position 2 (Fig. 1) and the second possibility in Eq. (7)]. Kirchhoff's laws give an additional equation governing the evolution of the shift voltage:

$$
\tau_{2} \dot{v}_{\mathrm{sh}}=k \bar{v}-v_{\mathrm{sh}},
$$

where 


$$
\tau_{2}=\frac{\omega_{\nu} C R_{B}}{N} \sqrt{\frac{I_{\mathrm{max}} R_{\mathrm{dc}}}{\widetilde{V}}}
$$

and $k$ is the gain of the amplifier (Fig. 1). In our experiments, $\tau_{2} \approx 6.9 \times 10^{3}$ for $C=530 \mu \mathrm{F}$. Thus the capacitor $C$ makes the evolution of $v_{\text {sh }}$ slow relative to node oscillations $\left(\dot{v}_{s h}\right.$ $\propto 1 / \tau_{2}$ ). Averaging Eqs. (6) over the ring, we find

$$
\ddot{\vec{v}}=\frac{1}{N} \sum_{n=1}^{N} F\left(\tau \dot{v}_{n}-v_{\mathrm{sh}}\right)-2 a \bar{v}+\frac{a}{k} v_{\mathrm{sh}} .
$$

The right hand side (rhs) of Eq. (28) includes a sum over all nodes, which complicates the analytical investigation of the dynamics. Below we derive the approximate equations using results from the preceding section for $\Phi$.

Introducing new variables

$$
x(t)=\frac{1}{T} \int_{t-T}^{t} \bar{v}(s) d s, \quad y(t)=\frac{1}{T} \int_{t-T}^{t} v_{\mathrm{sh}}(s) d s
$$

and using Eqs. (10), (24), (27), and (28), we get

$$
\begin{gathered}
\ddot{x}+2 a x=\Phi(y, m)+\frac{a}{k} y, \\
\dot{y}=\frac{1}{\tau_{2}}(k x-y) .
\end{gathered}
$$

In Eqs. (30), $y$ is a slow variable relative to the time scale of $x$. For each fixed value of $y\left(\tau_{2} \rightarrow \infty\right)$ in the 3D phase space $(x, \dot{x}, y)$, we have a fast Hamiltonian planar system with one steady state (center) at $[1 / 2 a p(y, m)+(a / k), 0]$. For a finite $\tau_{2}$, the slow motion along $y$ axis is accompanied by the fast oscillatory motion on $(x, \dot{x})$. Applying the averaging method [32], we obtain for the slow motion,

$$
\dot{y}=\frac{k}{2 a \tau_{2}}\left[\Phi(y, m)-\frac{a}{k} y\right] .
$$

Depending on the ratio $a / k$ and the properties of $\Phi$, Eq. (31) has up to seven steady states (for $N=6$ ). The origin ( $y$ $=0$ ) is always a steady state, which is stable for small amplifier gain:

$$
k<k^{*}=a / \Phi^{\prime}(0, N / 2) .
$$

To calculate $\Phi^{\prime}(0, N / 2)$, we use Eqs. (20) and (24):

$$
\Phi^{\prime}(y, m)=\frac{d F\left(w_{\text {up }}\right)}{d w_{\text {up }}} \frac{d w_{\text {up }}}{d v_{\text {sh }}} .
$$

Evaluating the derivative at the origin, we obtain

$$
\Phi^{\prime}\left(0, \frac{N}{2}\right)=-\left.\frac{d F}{d w_{\text {up }}}\right|_{w_{\text {up }}=1} .
$$

The rhs of Eq. (34) is linked to the local slope of the $I-V$ characteristics of the nonlinear resistor, where it crosses zero $(V=\widetilde{V})$. From Eq. (32), we have

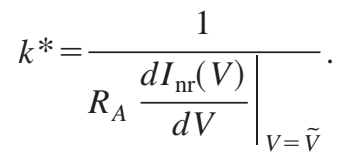

For the parameter values used in our experiments $\left(R_{A}\right.$ $=120 \mathrm{k} \Omega$ ) and the $I-V$ characteristics of the nonlinear resistor [30], we find $k^{*} \approx 0.08$. From the behavior of the optical mode, we experimentally estimate the value of $k^{*}$ by tuning $k$ while the origin remains stable. The result in this case is $k^{*} \approx 0.09$, close to the theoretical value. The other steady states, if they exist, are unstable for all parameter values. Thus if the origin is stable, it is globally stable. Hence, after a transient and corresponding mode jumps the optical mode $m=N / 2=3$ is excited with $v_{\mathrm{sh}}=0,\langle F\rangle=0$.

For a high amplifier gain, $k>k^{*}$, all steady states (including the origin) are unstable. Each mode is metastable (can exist only for some time, although long enough relative to the oscillation period) due to the continuous increase or decrease of the shift voltage. Thus the ring can choose a particular mode, say $l$, only temporarily since the change of the shift voltage leads to a bifurcation when the corresponding stable branch of the function $\Phi(y, l)$ (solid lines in Fig. 4) ends, with a subsequent jump to another branch corresponding to another mode. According to Eq. (31), the value $y$ (and, respectively, the shift voltage $\left.v_{\mathrm{sh}}\right)$ increases if $\Phi(y, m)$ $>a y / k$ and decreases otherwise. Hence, the number of modes involved in the mode rotation when modes in the ring successively replace each other depends on the ratio of $a / k$ and on the properties of the function $\Phi$. The change of direction, for instance from growth to decrease of $v_{\mathrm{sh}}$, happens at a jump when the new mode appearing in the ring has a value of $\Phi$ lower than $a y / k$ (below the nullcline). Figure 5 shows two experimental runs for different values of the amplifier gain $k$. In the region above the straight lines (nullcline) $\left\langle I_{\mathrm{nr}}\right\rangle=\left\langle V_{s h}\right\rangle /\left(R_{A} k\right)$, we have motion to the right and below to the left. The jumps from $m=3$ to $m=2$ in the upper panel and from $m=2$ to $m=1$ in the lower panel, labeled $A$ and ending at point $a$, lead to a change in circulation direction since they take the current below the nullclines for each run. The next change of circulation direction occurs at the jumps $B$ ending at point $b$. Thus we go clockwise in a loop through the ring visiting different branches by "jumping" between modes. In Fig. 5(a), for $k=0.38$ the loop does not include modes $m= \pm 1$, while these modes are included in the loop for $k=1$ [Fig. 5(b)], as theory predicts.

Figure 6 illustrates the behavior of a simulated ring with higher number of nodes $(N=8$ and $N=20)$. Note that several branches coincide for the two rings (we return to this in Sec. V).

We call the mode transition a jump. Actually the transient is finite, but very short compared to the lifetime of any mode. Figure 7 shows a transition (a jump) from $m=2$ to $m=1$. We calculated the mean current $\left\langle I_{\text {nr }}\right\rangle$ by averaging the current through the first node over a time equal to the interval 

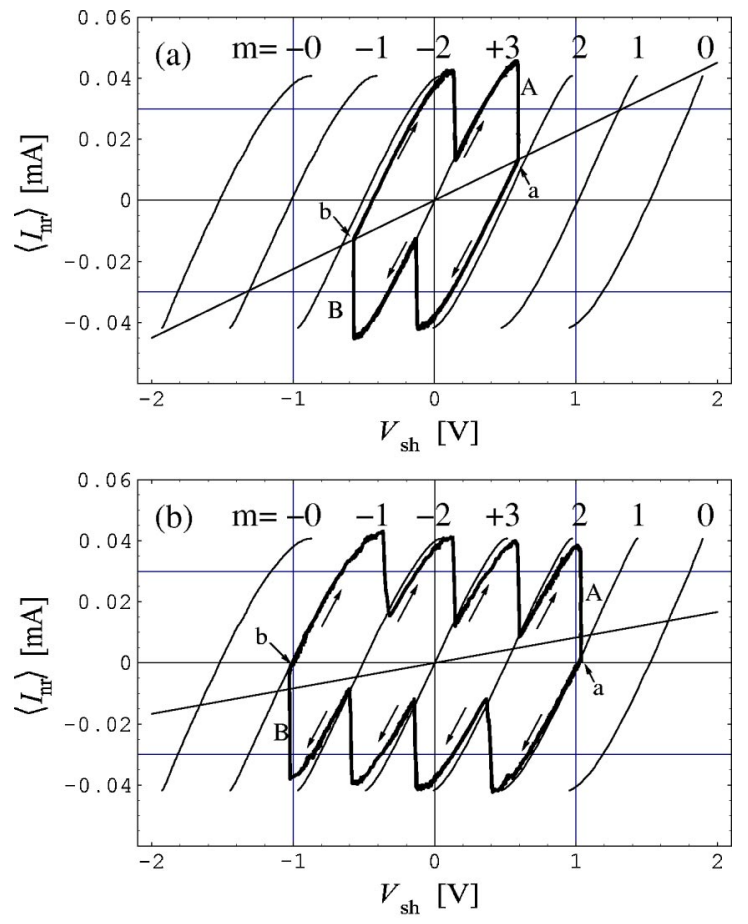

FIG. 5. Mode rotation; closed loops show experimentally obtained paths among quasistable branches of $\Phi$ (compare to Fig. 4) for two different values of $k$ : (a) $k=0.38$, three modes in the loop; (b) $k=1$, five modes in the loop. Straight lines with slope $\left(k R_{A}\right)^{-1}$ are nullclines.

between successive zeros of $\dot{v}_{1}(t)$. Strictly speaking, such averaging gives the mean value of the current only for a steady (established) oscillatory state [Figs. 7(a) and 7(c)], and is meaningless for the transient [Fig. 7(b)] where the procedure gives a curve linking the two stationary oscillatory states. However, the intermediate panel can be used to esti-

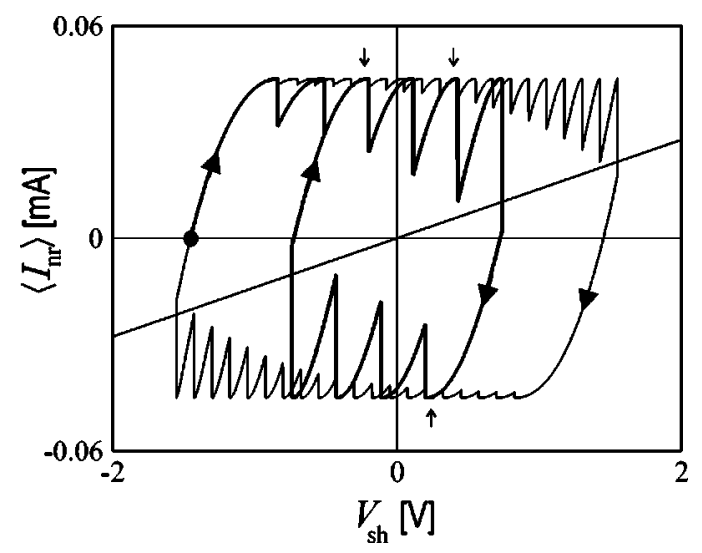

FIG. 6. Mode rotation and loops (numerical experiments); the thicker solid line corresponds to an 8-node ring and the thinner curve to a 20-node ring. The black dot indicates the starting point for both runs. The straight nullcline splits the plane into regions with opposite $\dot{V}_{\text {sh }}$. Small arrows mark three branches that are the same for the both rings (see text). Arrows on the branches show the direction of motion. The amplifier gain $k=0.6$ is higher than the critical value for both experiments.
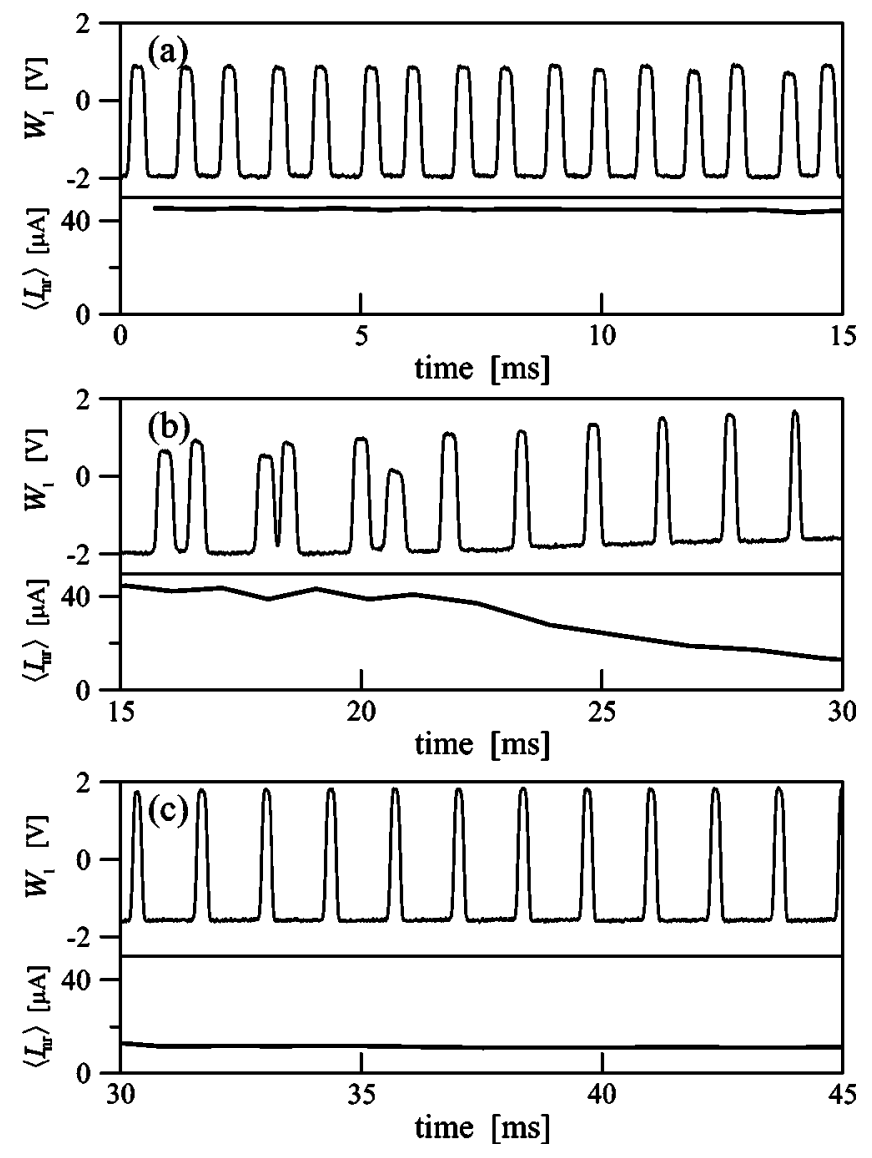

FIG. 7. Dynamical transition between modes $m=2$ and $m=1$, as the shift voltage $v_{\text {sh }}$ passes a critical (bifurcation) point. In each part, we show the voltage at the nonlinear resistor in the first node $\left(w_{1}=\tau \dot{v}_{1}-v_{\mathrm{sh}}\right.$ ) (upper curve), and the mean current (lower curve). (a) $m=2$ mode before jump, (b) transient, and (c) new $m=1$ mode after jump (compare to Fig. 4).

mate the duration of the transition. The transition from $m$ $=2$ to $m=1$ takes about $10 \mathrm{~ms}$ or eight oscillation periods of the $m=1$ mode. Hence it is very fast relative to the several second time scale for the shift voltage to make a loop, and even to the time interval between jumps (typically more than $0.3 \mathrm{~s}$ ).

\section{DISCUSSION}

We have investigated, both experimentally and theoretically, a Toda-Rayleigh ring with $N(N=6$ in experiments) identical nonlinear nodes, with exponential Toda-type interaction between nodes and Rayleigh-type dissipation-energy pumping mechanism (cubic nonlinearity with three regions such that the middle one has a negative slope and the other two have positive slopes).

In contrast to the original dissipationless Toda chain, our ring has a well-defined number of stable waves (including soliton like) which are selected by the energy balance condition: $(N-1)$ nonlinear oscillatory modes (waves) and two nonoscillatory ones. Oscillatory modes have different profiles and time scales. Each mode can be excited in the ring over some interval of shift voltage (Fig. 4). The analytical 
expression for stable branches of all $(N+1)$ different regimes of operation of the circuit agrees with the experimental observations. We have studied transitions between wave modes and proposed a suitable method to select modes independently of the initial conditions by varying a single parameter, i.e., the shift voltage. Moreover, by feedbacking the average voltage of the ring we have produced cyclic behavior, when different wave modes replace each other in the (periodic) loop. The number of modes visited in the cycle is chosen by changing the gain of the feedback circuit. Accordingly, our ring may be used, for instance, as a gait choosing tool for artificial locomotion. We have derived a simplified model that describes well the mode rotation.

Although our experiments use a rather short ring, the results may be applied to rings with an arbitrary number of nodes. Suppose that a stationary wave has been excited in the ring. Due to the geometry, several nodes may have the same oscillation phase. Assume that for mode number $m$, nodes $(n+\Delta n)$ and $n$ have the same phase. From Eq. (9), we have $\Delta n(m T / N)=j T$, or

$$
\Delta n=j \frac{N}{m}
$$

where $N /|m|$ denotes the wavelength that must be less than, or equal to the length of the ring and more than or equal to two nodes, and $j$ is an integer, which corresponds to the minimal number of lagging periods between nodes $n$ and $(n+\Delta n)$. Thus we have restrictions on the integers $m$ and $j$,

$$
1 \leqslant|j| \leqslant|m|<\frac{N+1}{2} \text {. }
$$

For all mode numbers and ring lengths, there exists at least one degenerate possibility, $\Delta n=N$, and consequently, $j$ $=m$. In this case, all nodes in the ring have different phases. When $N$ is a prime number, $j=m$ is the only solution satisfying Eqs. (35) and (36). Figure 8(a) illustrates this case for $N=2,3$, and 5, labeling different possibilities by two indices $N: m$. Node $(n+j)$ is closest in phase to nodes $n$, e.g., for $N=5$ in the configuration $5: 2$, the nearest in phase nodes are the next-nearest neighbors. Thus for rings with a prime number of nodes, we have $(N-1)$ oscillatory modes of this type.

If $\Delta n=N$ is not a prime number and $N$ and $m$ have no common factors, the fraction $\mathrm{N} / \mathrm{m}$ is irreducible, and again $j=m$. Figure $8(\mathrm{~b})$ for $6: 1,8: 1$, and 8:3 shows such configurations. In the latter case, nearest phases have third neighbors.

Let us consider the case $\Delta n<N$, which is realized when $N / m$ is reducible, i.e., $N$ and $m$ have a common factor $p$

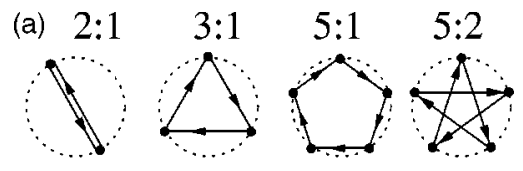

(b)
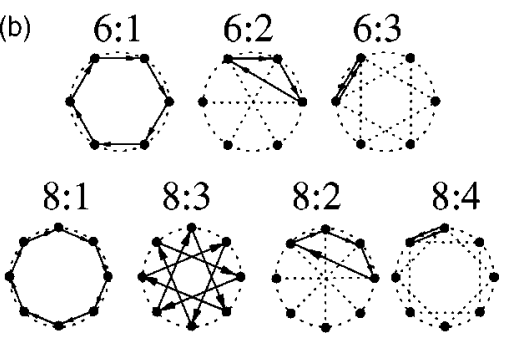

FIG. 8. Description of possible phase configurations, each case is labeled $N: m$, where $N$ is the number of nodes in the ring and $m$ is the mode number. Arrows join units with (unequal) nearest phases making clusters. Dotted lines join units with the same phase. (a) Basic configurations in rings with prime numbers of nodes (only the first three are shown). (b) Complex configurations that include clusters of small "rings" (two cases for $N=6$ and 8 are shown).

$\neq 1$. Accordingly, we can write $N=p N^{\prime}$ and $m=p m^{\prime}$. Equations (35) and (36) imply $j=m^{\prime}, \Delta n=N^{\prime}$, and $\left|m^{\prime}\right|<\left(N^{\prime}\right.$ $+1) / 2$. Hence, $m^{\prime}$ takes all possible values allowed in a ring with $N^{\prime}$ nodes and we can "split" the ring into $p$ symmetrically distributed clusters of smaller "rings" with $N^{\prime}$ nodes. Each such ring behaves like an $N^{\prime}$ ring. Oscillatory modes in each cluster will be the same as the corresponding modes in the $N^{\prime}$ ring. Figure 8(b) shows examples for $6: 2(p=2$ and $\Delta n=3)$; for $6: 3(p=3$ and $\Delta n=2)$; for $8: 2(p=2$ and $\Delta n=4)$; and for $8: 4(p=4$ and $\Delta n=2)$. Thus we obtain $(N-1)$ oscillatory modes for a ring of any length. Moreover, the stable branches for modes in the $N$ ring inherit the branches of all modes of the $N^{\prime}$ ring (Fig. 6 with $N=8$ and $N=20$ ) because Eq. (21) [and, accordingly, Eq. (24)] is invariant under the transformation $\left(N^{\prime}, m^{\prime}\right) \rightarrow\left(p N^{\prime}, p m^{\prime}\right)$. For example, in Fig. 6 two rings with $N=8$ and $N=20$ inherit branches from a ring with $N^{\prime}=4$ nodes ( $p=2$ and 5, respectively). Consequently, there are three branches (indicated by small arrows) corresponding to the oscillatory modes of a ring with four nodes that also belong to both long rings.

\section{ACKNOWLEDGMENTS}

Two authors (V.A.M. and W.E.) acknowledge the Ministry of Science and Technology of Spain for financial support. This research was supported by the same Ministry under Grant Nos. PB 96-599 and BFM2001-3723.
[1] E.R. Kandel, J.H. Schwartz, and T.M. Jessell, Principles of Neural Science, 4th ed. (McGraw-Hill, New York, 2000).

[2] H. Cruse, Trends Neurosci. 13, 15 (1990).

[3] H. Cruse, T. Kindermann, M. Schumm, J. Dean, and J. Schmitz, Neural Networks 11, 1435 (1998).
[4] G. Manganaro, P. Arena, and L. Fortuna, Cellular Neural Networks. Chaos, Complexity VLSI Processing (Springer-Verlag, Berlin, 1999).

[5] J. Ayers, J. Witting, N.J. McGruer, C. Olcott, and D. Massa, in Proceedings International Symporium on Aqua Biomecha- 
nisms, edited by T. Wu and N. Kato (Tokai University, 2000).

[6] P. Arena, L. Fortuna, and M. Branciforte, IEEE Trans. Circuits Systems I 46, 253 (1999).

[7] G. Schöner, W.Y. Yiang, and J.A.S. Kelso, J. Theor. Biol. 142, 359 (1990).

[8] J.J. Collins and I.N. Stewart, J. Nonlinear Sci. 3, 349 (1993).

[9] J.J. Collins and I.N. Stewart, Biol. Cybern. 68, 287 (1993).

[10] M. Golubitsky and I.N. Stewart, Arch. Rational Mech. Anal. 87, 107 (1985).

[11] Theory and Application of Coupled Map Lattices, edited by K. Kaneko (Wiley, Chichester, 1993).

[12] K. Kaneko and I. Tsuda, Complex Systems: Chaos and Beyond (Springer-Verlag, Berlin, 2001).

[13] G.R. Pradhan, N. Chatterjee, and N. Gupte, Phys. Rev. E 65, 046227 (2002).

[14] R. Carretero-Gonzalez, D.K. Arrowsmith, and F. Vivaldi, Phys. Rev. E 61, 1329 (2000).

[15] N. Gupte, A. Sharma, and G.R. Pradhan, Physica A 318, 85 (2003).

[16] D.G. Luchinsky, P.V.E. McClintok, and M.I. Dykman, Rep. Prog. Phys. 61, 889 (1998).

[17] F. Moss and P.V.E. McClintok, Noise in Nonlinear Dynamical Systems (Cambridge University Press, Cambridge, 1989), Vol. 3.

[18] E. del Rio, J.R. Sanmartin, and O. Lopez-Rebollal, Int. J. Bifurcation Chaos Appl. Sci. Eng. 8, 225 (1998).

[19] D. Ruswisch, M. Bode, D. Volkov, and E. Volkov, Int. J. Bi- furcation Chaos Appl. Sci. Eng. 9, 1969 (1999).

[20] E. Sanchez and M.A. Matias, Phys. Rev. E 57, 6184 (1998).

[21] See, e.g., M. Toda, Theory of Nonlinear Lattices (SpringerVerlag, Berlin, 1981).

[22] R. Hirota and K. Suzuki, Procs. IEEE 61, 1483 (1973).

[23] A.C. Singer and A.V. Oppenheim, Int. J. Bifurcation Chaos Appl. Sci. Eng. 9, 571 (1999).

[24] N. Islam, J.P. Singh, and K. Steiglitz, J. Appl. Phys. 62, 689 (1987).

[25] Y. Okada, S. Watanabe, and H. Tanaka, J. Phys. Soc. Jpn. 59, 2647 (1990).

[26] T. Kuusela and J. Hietarinta, Phys. Rev. Lett. 62, 700 (1989).

[27] V.I. Nekorkin and M.G. Velarde, Synergetic Phenomena in Active Lattices. Patterns, Waves, Solitons, Chaos (SpringerVerlag, Berlin, 2002).

[28] Lord Rayleigh, The Theory of Sound (Dover, New York, 1945), Vol. 1, Chap 3, p. 81.

[29] V.A. Makarov, W. Ebeling, and M.G. Velarde, Int. J. Bifurcation Chaos Appl. Sci. Eng. 10, 1075 (2000).

[30] V.A. Makarov, E. del Río, W. Ebeling, and M.G. Velarde, Phys. Rev. E 64, 036601 (2001).

[31] P. Horowitz and W. Hill, The Art of Electronics (Cambridge University Press, Cambridge, 1987).

[32] J. Guckenheimer and P. Holmes, Nonlinear Oscillations, Dynamical Systems and Bifurcations of Vector Fields (SpringerVerlag, New York, 1985). 